\title{
Diluted antiferromagnets in a magnetic field: evidence for a spin glass phase
}

\author{
K.D. Usadel and U. Nowak \\ Theoretische Tieftemperaturphysik and SFB 166, Universität Duisburg, Lotharstr. 1, 4100 Duisburg 1, Germany
}

\begin{abstract}
We have performed Monte Carlo simulations on diluted Ising antiferromagnets in the limit of high external magnetic fields $B$ and strong dilution. The two relevant lines in the $B-T$ plane of the phase diagram, $B_{\mathrm{c}}(T)$ above which antiferromagnetic long range order is no longer stable and $B_{\mathrm{eq}}(T)$ which marks the onset of hysteretic effects are determined. The random exchange-random field crossover exponent is obtained. The scaling behavior of $B_{\mathrm{eq}}(T)$ gives evidence for the occurrence of a spin glass phase.
\end{abstract}

Random field Ising systems and diluted Ising antiferromagnets in a uniform external magnetic field (DAFF) belong to the same universality class [1]. The phase diagram of the DAFF in the limit of strong disorder and high fields is still an open question and several possibilities have been discussed, like the existence of only a second order transition from antiferromagnetic order to paramagnetic behavior [2], the existence of a tricritical point with a crossover to a first order transition for low temperatures [3], the existence of a spin glass phase for low temperatures and high fields [4,5] and the existence of a stable domain state [6]. This frozen domain state which is usually thought to be metastable is a characteristic feature of the DAFF. It is obtained by cooling the system in an external field from the paramagnetic high-temperature phase. The mechanisms which are responsible for the corresponding hysteretic properties have been investigated experimentally [7] theoretically [8] and in computer simulations $[6,9]$.

The hamiltonian of the DAFF in units of the coupling constant $J$ reads

$H=\sum_{\langle i, j\rangle} \epsilon_{i} \epsilon_{j} \sigma_{i} \sigma_{j}-B \sum_{i} \epsilon_{i} \sigma_{i}$,

where $\sigma_{i}= \pm 1$ and $\epsilon_{i}=0,1$. Only nearest neighbour interaction is considered in our Monte Carlo simulation. The size of the system was $61 \times 61 \times 60$ with a dilution of $50 \%$.

In order to determine the phase diagram we computed the magnetization $M(T)$ and the internal energy $E(T)$ during field cooling $(\mathrm{FC})$ from the paramagnetic state and field heating $(\mathrm{FH})$ from the antiferromagnetic state (for details see ref. [9]). Due to the freezing of the system during FC there occur irreversibilities the onset of which marks $B_{\text {eq }}(T)$. Fig. 1 shows four of the computed $E(T)$ loops. The heating/cooling rate was $100 \mathrm{MCS} /$ point with a corresponding temperature interval of 0.01 . For low $B$ the energy of the system during $\mathrm{FH}$ is lower than during FC (upper curve). This behavior is reversed for higher fields where the domain state reached upon FC carries less internal energy (the two lowest curves). In the crossover region the difference between the energies of $\mathrm{FC}$ and $\mathrm{FH}$ procedure is too small to determine $B_{\text {eq }}(T)$ (see the loop for $B=1.2$ in fig. 1). Therefore we observed the corresponding magnetization loops which split in this region as well.

In comparing these two kinds of loops we have the possibility to determine $B_{\mathrm{eq}}(T)$ for all possible values of $B$. The resulting data are summarized in the phase diagram fig. 2 (triangles for the $M(T)$ data and crosses for the $E(T)$ data).

The second relevant line in the phase diagram is $B_{c}(T)$. In order to estimate this critical line we have looked for the maximum of $\partial M / \partial T$ during $\mathrm{FH}$ which marks the critical temperature in the crossover regime for random exchange to random field behavior [10], i.e. in the limit of small $B$. The corresponding data $T_{\mathrm{c}}(B)$ are shown as filled circles in fig. 2 .

As an estimate for the critical line in the limit $T \rightarrow 0$ we use the field where practically no difference in the energy of the FC and FH loops occurs. Here the

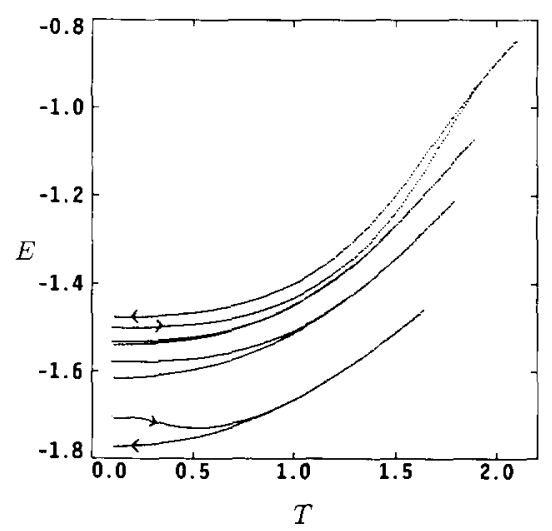

Fig. 1. Internal energy versus temperature during FH and FC for four different fields: $B=0.8,1.2,1.6,2.2$ (from above). 


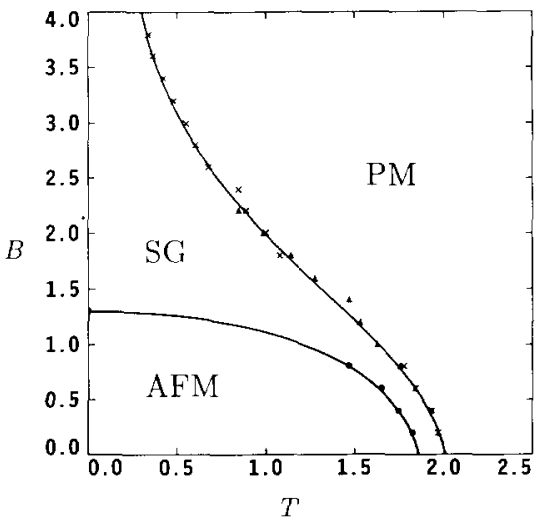

Fig. 2. Phase diagram of the DAFF for a dilution of $50 \%$ as explained in the text. The lines are guides to the eve.

stability of the long range ordered state vanishes and the domain state becomes stable for $B>B_{c}$. This point is marked in the phase diagram by a circle on the $B$-axis.

The phase diagram shown in fig. 2 is devided into three parts two of which easily can be identified: the high temperature paramagnetic phase and the low temperature antiferromagnetic phase which is stable for small fields. In order to further investigate the phase diagram we looked for the scaling behavior of $B_{\mathrm{cu}}(T)$ and $B_{\mathrm{c}}(T)$. Both of them are expected to scale with $\left(T_{\mathrm{N}}-c B^{2}-T\right)^{\phi_{\mathrm{ct}} / 2}$ for low fields [1] where $\Phi_{\mathrm{co}}$ is the crossover exponent from random bond to random field behavior. In the following we neglect the correction term $-c B^{2}$ which is expected to be small for low fields. Fitting the data for the lowest fields of $B_{\mathrm{c}}(T)$ to: $B_{\mathrm{c}}=a\left(T_{\mathrm{N}}-T\right)^{\phi_{\mathrm{cin}} / 2}$ we get the values $\Phi_{\mathrm{co}}=$ 1.41, $T_{\mathrm{N}}=1.88$ and $a=2.17$. At next we fitted the data of $B_{\mathrm{eu}}(T)$ in the same temperature region, the first seven points to $B_{\mathrm{eq}}=a\left(T_{\mathrm{eq}(0)}-T\right)^{\phi_{\mathrm{co}} / 2}$ obtaining the values $\Phi_{\mathrm{co}}=1.41 \pm 0.14, T_{\mathrm{eq}(\mathrm{l})}=2.01$ and $a=3.21$. As expected we get the same value of $\Phi_{c o}$ as for $B_{c}(T)$. This value is in very good agreement with experimental results $[7,10]$. In contradiction to experimental results $T_{\mathrm{e} 40}$ differs from $T_{\mathrm{N}}$. This of course is also obvious in fig. 2 and we attribute it to dynamical effects which will be discussed later.

As is obvious in fig. 2 there is a crossover to a new scaling behavior for higher fields. This leads us to fit the high field data, i.e. the last seven points to $\left(T_{\text {eq }}-\right.$ $T)^{\phi / 2}$, using the same value for $T_{\text {eq0 }}$ as before. We now get a new value of $\Phi=3.27 \pm 0.05$ which is just in between the theoretical value of $\Phi_{\mathrm{AT}}=3$ for the de
Almeida Thouless replica symmetry breaking line known from spin glass theory [11] and the experimental value $\Phi_{\mathrm{AT}}=3.4$ found recently in these systems [5].

Here some comments about the dynamical effects already mentioned earlier are in order. As is well known from spin glasses the position of an AT line depends on the cooling/heating rate which is used in experiments and simulations, respectively. In our simulations this effect could be observed also by calculating phase diagrams with $B_{\mathrm{cq}}(T)$ obtained through runs with different heating/cooling rates. The position of the AT line then changes as well as the value of $B_{\mathrm{cu}}(T)$ in the crossover regime but this does not affect the exponents $\Phi_{\mathrm{c} \text { o }}$ and $\Phi_{\mathrm{AT}}$. It is even this effect that explaines the difference between $T_{\Lambda}$ and $T_{\text {eq } 9}$. In the limit of very long cooling/heating rates $T_{\text {(eq) }}$ tends to $T_{\mathrm{N}}$. The phase diagram which is shown here is that we obtained for the slowest rate.

To summarize, our phase diagram is in good agrecment with refs. $[5,4]$. We conclude that the domain state is a stable phase between the AT line and $B_{\mathrm{c}}(T)$ with the characteristics of a spin glass, although the spin glass behavior here is field induced. Without an external field the system is long range ordered and there is no frustration.

\section{References}

[1] S. Fishman and A. Aharony. J. Phys. C 12 (1979) L729.

[2] A.T. Ogielski and D.A. Huse. Phys. Rev. Lett. 56 (1986) 1298.

[3] H.T. Diep, S. Galam and P. Azaria, Europhys. Lett. 4 (1987) 1067.

[4] 1. Korenblit and E. Shender, Sov. Phys. JETP 62 (1985) 1030. J.R.L. de Almeida and R. Bruinsma, Phys. Rev. B 35 (1987) 7267.

[5] F.C. Montenegro, U.A. Leitāo, M.D. Coutinho-Filho and S.M. Rezende, J. Appl. Phys. 67 (1990) 5243. F.C. Montenegro, A.R. King, V. Jaccarino, S.-J. Han and D.P. Belanger, to be published.

[6] G.S. Grest, C.M. Soukoulis and K. Levin, Phys. Rev. B 33 (1986) 7659.

[7] U.A. Leitão, W. Kleemann and I.B. Ferreira, Phys. Rev. B 38 (1988) 4765. P. Pollak, W. Kleemann and D.P. Belanger, Phys. Rev. B 38 (1988) 4773.

[8] J. Villain. Phys, Rev. Lett. 52 (1984) 1543.

[9] U. Nowak and K.D. Usadel, Phys. Rev. B 39 (1989) 2516.

[10] W. Kleemann, A.R. King and V. Jaccarino, Phys. Rev. B 34 (1986) 479.

[11] J.R.L. de Almeida and D.J. Thouless, J. Phys. A 11 (1978) 983. 